\title{
FACTORS AFFECTING USER PREFERENCE FOR MOBILE VIDEO QUALITY
}

\author{
Andreea Molnar \\ University of Portsmouth \\ School of Creative Technologies \\ Winston Churchill Ave, Portsmouth, United Kingdom \\ andreea.molnar@port.ac.uk
}

\begin{abstract}
People increasingly use their mobile phones and mobile data to access different media types, including video. Due to mobile phone limitations, but also due to the scarce bandwidth on mobile networks, video content is often adapted to fit with these requirements by reducing its quality. In this study we look into how the user preference for a certain multimedia content (e.g. video, audio, text and images or only text) and the usage of mobile data affects user preference for video quality. The results show a statistically significant difference in the video quality preference only for participants who reported that video is one of the preferred multimedia content types, and the ones who did not. We also discuss how these results could be used for multimedia streaming and in telecommunications marketing research to improve user experience and increase network operators and content provider revenues.
\end{abstract}

Index Terms - Consumer behaviour, multimedia, video adaptation, video quality

\section{INTRODUCTION}

The adoption of mobile phones is continuously increasing and more people are using smartphones due to their increased affordability [1]. This led to increases in mobile data traffic and especially in video traffic [2] that has reached 64\% in 2014 and it is estimated to reach $80 \%$ of all consumer data traffic by 2019 [3]. As mobile network resources are scarce, research and practice emphasize the importance of adaptive video delivery as a means of dealing with scarce bandwidth [4,5] and with the data caps that are put in place by mobile operators to impede congestion and increase revenues $[6,7]$. Increases in mobile traffic are likely to continue to strain mobile network resources even with the introduction of higher bandwidth networks [8]. Moreover, mobile phones are sometimes not able to support the best quality available and the video needs to be adapted to a quality that can be displayed on mobile phones.

Although there is substantial research on the advantages of adaptation (e.g. congestion avoidance, increasing the number of customers served on the same bandwidth, reducing costs, battery consumptions) $[6,9,11,12]$ the relationship between people's preferences and video quality is still not well understood; this relationship is highly complex and sometimes counter intuitive. A better understanding of users' preferences will help not only in providing better video adaptation solutions and end user video services, but will also improve our understanding on how to market existing video services. This has the potential of improving user satisfaction with such services, which has been shown to improve consumer loyalty [13]. Moreover, it could lead to an increase in network and content provider revenues through: (a) increasing consumer quality of experience and their loyalty; and (b) a better management of existing bandwidth and traffic to the servers by optimising adaptive video streaming algorithms.

In this article, we aim to improve the understanding of people's preference for different video quality levels by investigating whether the preference for certain multimedia content (e.g. video, audio, text and images or only text) and previous use of mobile data influence user preferences for a certain video quality. The rest of this article is organised as follows. The next section briefly introduces the related work, focusing on the user adoption and user experience with mobile data and the factors affecting video quality preferences. The article continues by presenting the study set up and the design procedure and analysis followed by the study's findings. The following section discusses the findings and presents the implications of the study. The article ends with our conclusions and planned future work.

\section{LITERATURE REVIEW}

This research draws from user adoption and interaction under different mobile data and factors affecting video quality preferences. A brief introduction of research in these areas is presented below.

\subsection{Mobile Data Adoption and User Experience with Mobile Data}

Existing research on user adoption and user experience with mobile data has focused on how people use the mobile In- 
ternet and what factors influence its adoption. For example, [14] showed that innovativeness, low desire for social contact and technology optimism influence consumer acceptance and affects the behaviour with regards to the usage of the mobile Internet. They have also found that gender affects consumer choices of mobile Internet. The study presented in [15] showed that the usage of mobile services (e.g. GPS via the mobile Internet) is not significantly affected by the means through which users subscribe. They have also found that demographic data (consumer gender, age, occupation and income) have a significant effect on the type of contract the user will adopt (i.e. voice and $3 \mathrm{G}$ Internet and $3 \mathrm{G}$ Internet fee).

Other studies have looked into how mobile data billing plans affect the user experience. They have found that cost is one of the factors influencing the user experience when accessing the Internet from mobile phones [16, 17] and one of the factors affecting the usage of data from smartphones $[18,19]$. People change their mobile data usage depending on the billing plan used (e.g. to optimise the time spent on the Internet if the billing plan is capped) [17]. Moreover, people own past experiences or highly publicised stories that report high mobile billing plans which are likely to have an effect on how people use and interact with mobile data [16]. Mobile Internet billing plans in which the user is paying based on the quantity of data consumed are hard to understand for the users: the users will not always understand or find it easy to estimate how much data they consume [17].

\subsection{Factors Affecting Video Quality Preferences}

Several studies have looked at user preferences for different video quality and what factors affect them [20, 21, 22, 23]. [20] show that the user preference for a certain video quality changes depending on the billing plan s/he has (unlimited or capped billing plan). In the case of capped billing plans, the usage preference is also influenced by the consequences of exceeding the user cap (i.e. whether the user can buy another bundle or s/he has her/his bandwidth throttled) [20]. Moreover willingness to pay for the video quality might be explained by the user attitude towards risk [21]. Cultural and personality traits (i.e. openness, conscientiousness, extraversion, agreeableness and neuroticism) also have an influence on perception of multimedia quality [23]. [22] have looked at factors that affect enhanced video quality preferences and uncovered that not all the participants liked video enhancement. The participants could be broadly divided in two groups: those who like and those who dislike enhancements [22]. The preference for video enhancement have been shown to be dependent on the video content type [22]. The study [22] has also found a preference for human faces to be less enhanced.

\subsection{Conclusions}

All the studies presented above have assessed different factors affecting user adoption of mobile data $[14,15]$, or looked into how different billing plans affect user satisfaction and interaction with mobile data $[16,17]$ but have not looked into how they affect user preference for video quality. Research that has assessed how different factors affect video quality has addressed the influence of video content type and enhancement [22] and how certain billing plans [20, 24], user attitude towards risk [21] or cultural traits [23] affect user preference for video quality. As opposed to the above studies, in this article we focus on assessing whether the user preference for a certain multimedia content or its previous usage of mobile data affects the preference for a certain type of video content. We also look at the effect of demographic factors (i.e. age and gender) have on video quality preference.

\section{STUDY}

\subsection{Sample}

A total of 76 people took part in the study on a volunteering basis. More men (74\%) took part in the study than women (36\%). The age of the participants varied from 19 to 57 years old, with an average age of 31 years old. None of the participants were working in video quality assessment.

\subsection{Design and Procedure}

A quasi-experimental study [25] was organised. During the study the participants were first asked to fill in a survey through which demographic information, people's preference towards a certain multimedia content and the usage of mobile Internet were collected. Afterwards the participants had to watch five video clips on a Google Nexus smartphone (resolution: $800 \times 480$, screen size: 3.7", internal memory $512 \mathrm{MB}$, CPU $1 \mathrm{GHz}$ ). They were asked to select their preferred video version (i.e. what video they would prefer to watch) among the five presented. The participants were not asked to rate the video quality on the MOS scale. The participants were instructed that they could view the video clips as many times as they wish before making the decision but they had to see them all at least once. All the participants viewed the video clips from the same mobile device. The video clips were shown to the participants in a random order to avoid bias.

The encoding of the original video content used for the study was MPEG-4 AVC/H.264. The video content was selected in such a way that it will show various levels of dynamicity and contain also animated material. The five versions of the same video clip with different encodings were used in this study. The video clips were embedded in a web page.

The videos have different quality levels, the resolution and/or bitrate being affected during the adaptation as presented in Table 1 . The videos were around one minute and 


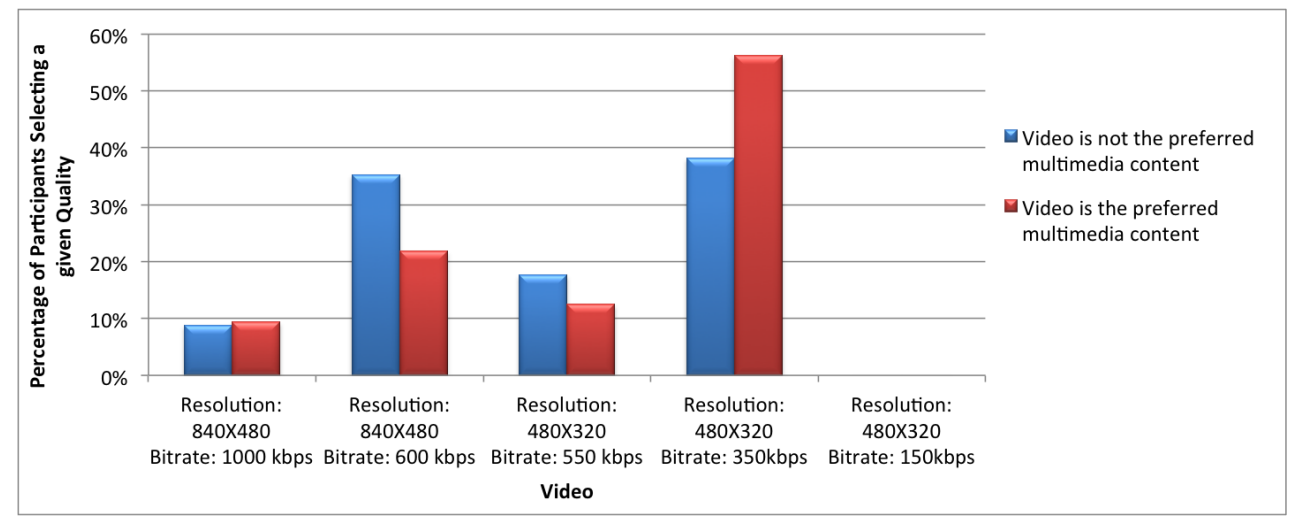

Fig. 1. Percentage of participants preferring a certain video quality depending on whether video content is or not their preferred content to be accessed through a mobile device

Table 1. Resolution, Mean Opinion Score (MOS) and bitrate for the video versions used in the evaluation

\begin{tabular}{|c|c|c|}
\hline Resolution & MOS & Bitrate (kbps) \\
\hline $840 \times 480$ & 5 - Excellent & 1000 \\
$840 \times 480$ & 4 - Good & 600 \\
$480 \times 320$ & 3 - Fair & 550 \\
$480 \times 320$ & 3 - Fair & 350 \\
$480 \times 320$ & 2 - Poor & 150 \\
\hline
\end{tabular}

thirty seconds. The content was transcoded using XMedia Recode [26]. The videos were encoded MPEG-4 and have a frame rate of $29.97 \mathrm{fps}$, their original frame rate. These five quality levels were used in previous studies and selected based on industry recommendations [21]. The MOS values presented in Table 1 were obtained by computing Structural Similarity index [27] and mapping the results on the MOS scale as described in [28]. The participants were not provided the information presented in Table 1 but they were made aware that the videos presented are of different qualities.

\subsection{Data Manipulations and Analysis}

The variables (multimedia, text+image, audio, text, usage of mobile Internet and usage of video over mobile Internet) were operationalized using a dichotomous scale (1=yes; $0=$ no) based on whether the respondent had the given multimedia as his preferred content or has used or not the mobile Internet and video over the mobile Internet. The user preference for a given quality level was operationalized using a nominal scale (1 to 5) depending on the quality the user selected as described below:

- 1 was used to operationalize the highest video quality provided to the participants: the video clip having the resolution of $840 \times 480$ and encoded at $1000 \mathrm{kbps}$
- 2 was used to operationalize the video clip having the resolution of $840 \times 480$ and encoded at $600 \mathrm{kbps}$

- 3 was used to operationalize the video clip having the resolution of $480 \times 320$ and encoded at $550 \mathrm{kbps}$

- 4 was used to operationalize the video clip having the resolution of $480 \times 320$ and encoded at $350 \mathrm{kbps}$

- 5 was used to operationalize the lowest video quality provided to the participants: the video clip having the resolution of $480 \times 320$ and encoded at $150 \mathrm{kbps}$

The Student t-test [29] was used to measure the differences between two groups and a 95\% confidence interval was considered for statistical significance. Pearson productmoment correlation coefficient [30] was used to determine whether there is any correlation between demographic variables (age and gender) and participants preference for a certain video quality.

\subsection{Findings}

Multimedia Preference: A statistically significant difference was obtained between the participants who reported that video is one of the preferred multimedia content types on mobile phones and the ones who did not ( $\mathrm{p}=0.049, \mathrm{~F}=4.238$ ). The persons who selected video as their preferred multimedia content selected lower video quality than the ones who did not select that they prefer it (see Fig. 1 for the percentage of the participants selecting a video of a certain quality level). The participants were almost equally divided between those who prefer video content on mobile phones $(49 \%)$ and those who do not $(51 \%)$.

The participants reported no statistically significant difference in preference for video quality. The same was was obtained for audio ( $\mathrm{p}=0.124, \mathrm{~F}=2.514)$ or text and images $(\mathrm{p}=0.630, \mathrm{~F}=0.238)$, although users that did not select audio 


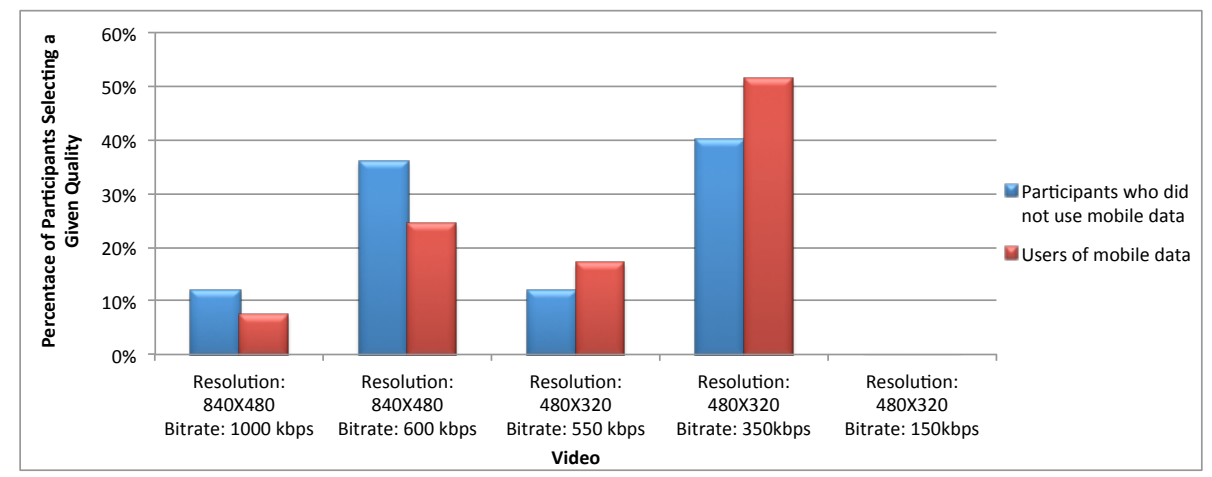

Fig. 2. Percentage of participants preferring a certain video quality for the users and non users of mobile data

Table 2. Differences obtained between participants' preference for multimedia content and video quality

\begin{tabular}{|c|c|}
\hline Mobile & $\begin{array}{c}\text { Statistical } \\
\text { Significant } \\
\text { Multimedia Preference }\end{array}$ \\
Difference \\
\hline Video - Other media types & Yes \\
Audio - Other media types & No \\
Text and Images - Other media types & No \\
Text - Other media types & No \\
\hline
\end{tabular}

or text and image as their preferred option selected a slightly higher video quality. In the case of people who selected text only, they selected a higher quality than the ones who said that they did not prefer text, although the difference was not statistically significant for text only $(\mathrm{p}=0.898, \mathrm{~F}=0.017)$.

Table 2 summarises the results presented in the above paragraphs. The only media type for which a statistical significant difference was obtained in people preference for video quality was between the participants who reported video as their preferred media content to watch on their mobile phones and those who did not.

Mobile Data: Although people who did not use mobile data before selected better quality than those who used mobile data (see Fig. 2 for the percentage of participants selecting a certain video quality), no statistically significant difference in their preference for video quality, was obtained between those who used mobile data in general and those who did not $(p=0.460, F=0.562)$ or between those who use mobile data to access video content and those who do not $(\mathrm{p}=0.35)$. In our sample, there were less participants that did not use mobile data $(38 \%)$ than those who use mobile data $(62 \%)$.

Gender and Age: We have also assessed the effects of demographic variables on multimedia preferences. The results showed no correlation between gender and video preference for multimedia quality $(\mathrm{r}=0.092, \sigma=0.431)$ at 0.01 level, but it shows statistical significant weak positive corre- lation between age and video preference for multimedia quality $(\mathrm{r}=0.299, \sigma=0.009)$ at the same level. Fig. 3 presents the percentage of each participants selecting a given video quality based on their gender.

\section{GENERAL DISCUSSIONS AND IMPLICATIONS}

This article expands on existing research that looks into how user preferences and usage of mobile data affect her/his choice for video quality. It provides a better understanding on how user preference for video quality may be influenced by the user preference for video content. Similarly with previous research [20], we found either no correlation, or weak correlation between demographic variables (i.e. age and gender) and video preference towards a certain video quality.

This research contributes to a better understanding of user preference towards different quality levels and opens up new directions for technical support and design of mobile video streaming systems. It has implications that advance the field of marketing research with respect to people's adoption of multimedia content on mobile devices. This study can help brands to segment and target consumers based on preferences for multimedia quality.

The study shows that users who did not select video content as their preferred media to watch on mobile, are more likely to prefer a higher quality multimedia content than the ones who did. It is possible that users that consume video content on mobile devices have lower expectations for video quality than those who did not, because they are aware of the mobile phones and network limitations in displaying high quality content.

As optimising the user experience with adaptive video delivery is still a challenge to date, this study could help guide and improve existing video adaptation algorithms. This has implications on network operators, providers and consumers. Network operators could benefit by having lower congestion levels and being able to serve more customers. Content providers could reduce the traffic at their servers through the 


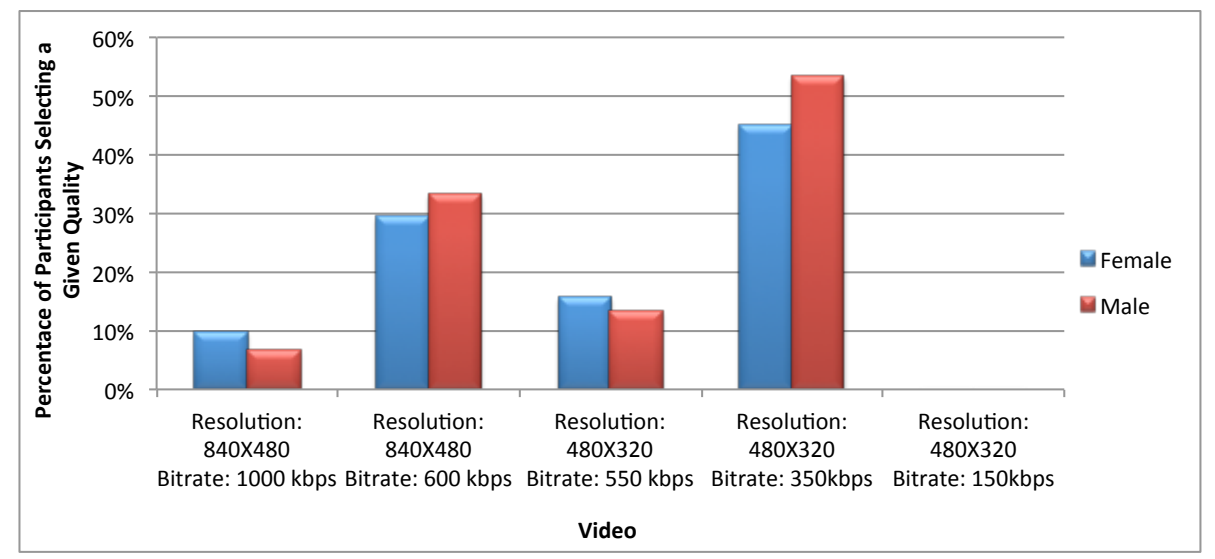

Fig. 3. Percentage of participants preferring a certain video quality based on their gender

adaptation. Adaptation might also lead to price reduction for the content providers who are subsidising the users' cost of mobile data. As the users afford to consume more data, content providers who (a) charge users per unit of content consumed (e.g. Vimeo) or (b) make their revenue through advertisements, advertisements that increases proportional with the amount of content consumed, could increase their revenues proportionately. Both network operators and content providers will benefit from increased customer satisfaction, as it leads to an increase in customer loyalty [13]. This would lead to a win-win situation for content providers, network operators and consumers.

There are some limitations of the study worth noting. We use a relatively small sample of videos and participants. Using multiple videos might show different results depending on the video used. Our sample size has more men than women and this might have also affected the results. The study was conducted in only one country - and as cultural traits might affect preference for video quality [23], performing this study in a different country might also lead to different results.

\section{CONCLUSIONS AND FUTURE WORK}

This article presented the results of a study assessing how the user preference for different media types and the use of mobile data affect user preference for video quality. The results showed that the participants who preferred video content were more likely to select a lower video quality compared to the ones who did not reported video as their preferred media content on mobile devices. This is the only media type (among the four considered: video, audio, text and images, and text only) for which a statically significant difference was obtained. No statistically significant difference in video quality preference was obtained between the participants who use mobile data and those who do not. The results also show a weak positive correlation between age and preference for video quality.

Future work will aim to validate these results across more video qualities, different video content types and across multiple devices. We also want to consider other factors that could influence the user preferences such as the quality of the network connection, the context in which the user is, user preferences for the given content and cultural differences that may arise. Ultimately we want to propose a video adaptation mechanism that would consider the above factors as well as other factors that existing research has shown to influence user preference. We are planning to assess the proposed mechanism and determine how it affects the user quality of experience.

\section{REFERENCES}

[1] emarketer, "Smartphone users worldwide will total 1.75 billion in 2014," Available at http://www.emarketer.com/Article/Smartphone-UsersWorldwide-Will-Total-175-Billion-2014/1010536.

[2] I. Trestian, S. Ranjan, A. Kuzmanovi, and A. Nucci, "Taming the mobile data deluge with drop zones," IEEE/ACM Transactions on Networking (TON), vol. 20, no. 4, pp. 1010-1023, 2012.

[3] CISCO, "Cisco visual networking index: Forecast and methodology, 2014-2019," 2015, Available at http://www.cisco.com/c/en/us/solutions/collateral/serviceprovider/ip-ngn-ip-next-generationnetwork/white_paper_c11-481360.html.

[4] R. Garrido-Cantos, J. De Cock, J.L. Martinez, S. Van Leuven, P. Cuenca, and A. Garrido, “Temporal video transcoding from h. 264/avc-to-svc for digital tv broadcasting," Telecommunication Systems, pp. 1-21, 2015. 
[5] I. King, "For carriers, video optimization can be an answer to "mobile warming," 2012, Available at http://www.atelier.net/en/trends/articles/carriersvideo-optimization-can-be-answer-mobile-warming.

[6] A. Molnar and C.H. Muntean, "Cost-oriented adaptive multimedia delivery," Broadcasting, IEEE Transactions on, vol. 59, no. 3, pp. 484-499, 2013.

[7] J. Newman, "Got bandwidth caps? netflix has you covered," 2011, Available at http://www.technologizer.com/2011/06/23/gotbandwidth-caps-netflix-has-you-covered/.

[8] I. Chih-Lin, C. Rowell, S. Han, Z. Xu, G. Li, and Z. Pan, "Toward green and soft: a $5 \mathrm{~g}$ perspective," Communications Magazine, IEEE, vol. 52, no. 2, pp. 66-73, 2014.

[9] F. Kong, X. Lu, M. Xia, X. Liu, and H. Guan, "Distributed optimal datacenter bandwidth allocation for dynamic adaptive video streaming," in Proceedings of the 23rd Annual ACM Conference on Multimedia Conference. ACM, 2015, pp. 531-540.

[10] S. Petrangeli, J. Famaey, M. Claeys, S. Latré, and F. De Turck, "Qoe-driven rate adaptation heuristic for fair adaptive video streaming," ACM Trans. Multimedia Comput. Commun. Appl., vol. 12, no. 2, pp. 28:1-28:24, Oct. 2015.

[11] C. Singhal, S. De, R. Trestian, and G.-M. Muntean, "ewu-tv: User-centric energy-efficient digital tv broadcast over wi-fi networks," Broadcasting, IEEE Transactions on, vol. 61, no. 1, pp. 39-55, 2015.

[12] W. Jung and Y. Kwon, "Differences between lte and 3g service customers: Business and policy implications," Telematics and Informatics, vol. 32, no. 4, pp. 667-680, 2015.

[13] J. Koenigstorfer and A. Groeppel-Klein, "Consumer acceptance of the mobile internet," Marketing Letters, vol. 23, no. 4, pp. 917-928, 2012.

[14] M.-H. Hsiao and L.-C. Chen, "Smart phone demand: An empirical study on the relationships between phone handset, internet access and mobile services," Telematics and Informatics, vol. 32, no. 1, pp. 158-168, 2015.

[15] P. Isomursu, R. Hinman, M. Isomursu, and M. Spasojevic, "Metaphors for the mobile internet," Knowledge, Technology \& Policy, vol. 20, no. 4, pp. 259-268, 2007.

[16] V. Roto, R. Geisler, A. Kaikkonen, A. Popescu, and E. Vartiainen, "Data traffic costs and mobile browsing user experience," in Proceedings of the 4th MobEA Workshop on Empowering the Mobile Web, Edinburgh, UK, 2006, pp. 1-6.
[17] A. Molnar, "On better understanding the usage of mobile phones for learning purposes," Bulletin of the IEEE Technical Committee on Learning Technology, vol. 16, no. 2/3, pp. 18, 2014.

[18] A. Kumar, R. Shankar, and R.M. Debnath, "Analyzing customer preference and measuring relative efficiency in telecom sector: A hybrid fuzzy ahp/dea study," Telematics and Informatics, vol. 32, no. 3, pp. 447-462, 2015.

[19] A. Molnar, "Video quality vs. mobile data billing plans," Telematics and Informatics, vol. 33, no. 1, pp. 227-231, 2016.

[20] A. Molnar and C. Hava Muntean, "What can people risk attitude tell us about people preference for multimedia quality?," Information Technology \& People, vol. 28, no. 2, pp. 383-404, 2015.

[21] P.M. Satgunam, R.L. Woods, P.M. Bronstad, and E. Peli, "Factors affecting enhanced video quality preferences," Image Processing, IEEE Transactions on, vol. 22, no. 12, pp. 5146-5157, 2013.

[22] M.S. Scott, S.C.Guntuku, Y. Huan, W. Lin, and G. Ghinea, "Modelling human factors in perceptual multimedia quality: On the role of personality and culture," in Proceedings of the 23rd Annual ACM Conference on Multimedia Conference. ACM, 2015, pp. 481-490.

[23] A. Molnar and C. Muntean, "Educational content delivery: An experimental study assessing student preference for multimedia content when monetary cost is involved," in Intelligent Systems Design and Applications (ISDA), 2010 10th International Conference on. IEEE, 2010, pp. 871-876.

[24] T.D. Cook, “Quasi-experimental design," Wiley Encyclopedia of Management, 2015.

[25] S. Dörfler, "Xmedia recode," 2015.

[26] R. Dosselmann and X.D. Yang, "A comprehensive assessment of the structural similarity index," Signal, Image and Video Processing, vol. 5, no. 1, pp. 81-91, 2011.

[27] T. Zinner, O. Abboud, O. Hohlfeld, T. Hossfeld, and P. Tran-Gia, "Towards qoe management for scalable video streaming," in 21th ITC Specialist Seminar on Multimedia Applications-Traffic, Performance and QoE, Miyazaki, Japan, 3 2010, pp. 64-69.

[28] T.R. Black, Doing quantitative research in the social sciences: An integrated approach to research design, measurement and statistics, Sage, 1999.

[29] I. Lawrence and K. Lin, "A concordance correlation coefficient to evaluate reproducibility," Biometrics, vol. 45, no. 1, pp. 255-268, 1989. 